\title{
Intellectual Green Corridor for Crisis Wellbeing Transference
}

\author{
Inbalatha.K, Palaniswamy K.M
}

\begin{abstract}
Traffic is a major concern for most of the metropolitan cities of the world. The design proposes a notion for Traffic Control System which is more imaginativethan currently existing schemes.The system automatically affords a distinctive lane in which entirely the red signal indication will be turned spontaneously to green intended for the ambulance. Subsequently this assists the ambulance instantly in reaching its destination within stint.In accumulation to the Traffic Control Scheme, wellbeing specialist care Scheme displays the patient long-suffering state of affairs resembling heartbeat, blood pressure. This system comprises of dedicated intellectual smart ambulance with GPS, GSM and smart mobile solicitation beside with Internet of Things (IoT).The patient's state of affairs will be directed to the medical wingover cloud.The information of the patient is sent to the hospital via GSM module and blood bank gets information only if the condition is chosen as serious. The projected effort stays targeted to plan and progress an operative traffic control scheme for smart ambulance. The outcomes of the recommended traffic control model transports upright decline of time by clearing the traffic very fast and protect the patient's lifespan at the most primitive.
\end{abstract}

Keywords: Blood pressure, Internet of Things, Metropolitan, Smart Ambulance.

\section{INTRODUCTION}

In crisis situation, every single instant is essential in saving an individual's life. Researchers and traffic engineers have developed several traffic signal priority strategies for EVs to pass intersections quickly and safely[1]. The objective of the efficient effort is to custom the every second resourcefully to rescueindividual. Nowadays countless lives are being perishedbeforehand the onegrasps the hospital in ambulance or due to shortage of basic data about the state of the patient and the hindrance caused due to this. The proposed work is designed to hoard the life at the most primitive.

The foremost endeavor of the mission is that while the patient is in ambulance in urgentsituation the ambulance should arrive the hospital utmost fast and to driveall necessary information and state about the patient to hospital for the earlier preparations for the treatment. The traffic control system provides a unique corridor in which entirelyred signal indication will be turned spontaneously to green intended for the ambulance. Subsequently this assists the ambulance instantly in reaching its destination within stintby which that patient gets needed treatment on time along with this system patient parameters are also measured. In the currentmethod we don't have programmedmechanism traffic control, in arrears to this ambulance is not able to get in touch with the hospital earlier.

Revised Manuscript Received on September 22, 2019.

Inbalatha.K, Assoc. Prof, Dept. of ECE, Dr.T.Thimmaiah Institute of Technology, KGF,inbalathaphd@gmail.com

Palaniswamy K.M, Professor, Dept. of ECE,Dr.T.Thimmaiah Institute of Technology, KGF,mail2kmp@gmail.com
The method includes effectual smart ambulance with GPS, GSM and smart phone application like Blynk through IoT. The previous works to monitor patient's conditions were performed using Micro- Electro Mechanical System (MEMS) and Bluetooth communication was used to transfer the data[2]. In the proposed system the patient's factors will be examined in ambulance and the information will be directed to the nearest hospital, at that juncture ambulance will decide on the lane to hospital and traffic signal in this direction will be turned to green using RFID reader and RFID tag, microcontroller changes the state of the signal.Heart Rate measurement is done using photoplethysmographymethod. LM-35 is used to measure the patient's body temperature and these parameters were sent to a PC in ambulance via serial communication besides this data was sent to the hospital server, in additionin traffic control part an RF transmitter on the ambulance was communicated with the RF receiver mounted on the signal post [3].With emergency vehicle clearance, the traffic signal turns to green as long as the emergency vehicle is waiting in the traffic junction. The signal turns to red, only after the emergency vehicle passes through [4].Often the patient's parameters will be directed to the hospital to get the ideas from the specialists in the hospital to supervise patient's situation. This venture is embattled to plan and progress a real-time intellectual emergency conveyance model.

\section{WORKS REVIEW}

In the period of smart metropolises, citizens face various difficulties concerning wellbeing issues like not receiving treatment on time. 'Green Corridor' is the model by which patient will get the required treatment on instant [5]. In sensible motorcar completely different sensing elements like pulse sensor, blood pressure, ECG will be judging condition of the essence parameters, the status of these parameters will be send to hospital's catalog, concurrently traffic signals will be operated by using GPRS communication through cloud. As the smart ambulance will get into the range of $100 \mathrm{~m}$, in which entirely the red signal indication will be turned spontaneously to green intended for the ambulance. The communication will be done by GPRS through cloud.A two-level strategy at signalized intersections for preventing incident-based urban traffic congestion by adopting additional traffic warning lights [6].

Intelligent traffic control method considers the priority for crisis conveyances based on the kind of an incident and a process to detect and respond to the hack of traffic signals that have been proposed [7]. An experiment employing a simulation software package, namely Simulation of Urban Mobility (SUMO) was conducted. Researchers discussed about the process to reduce traffic congestion by making 
one signal green for emergency vehicles.

For further development of above methods, a concept called 'Traffic Control System for Smart Ambulance" describes the monitoring of wellbeing parameters accessed by different sensors deployed on patient's body and transferring these to hospital system. At the equal instant traffic signal indications are controlled to enter the hospital as in the early hours as probable

\section{HARDWARE IMPLEMENTATION}

\section{Traffic Control System}

The traffic management system is consider as one of the major dimensions of a smart city. With the rapid growth of population and urban mobility in metropolitan cities, traffic congestion is often seen on roads [8]. The Radio frequency identification(RFID) transceiver is a device comprising both a transmitter and a receiver. Communicates with a passive tag. The Tag is placed in the ambulance. Passive identifiers have no power supply of their individual and as an alternative their develop power from the instance magnetic attraction field. Usually, at the epicenter of every identifier might be a chip. When the tag arrives at the generated RF field, it is capable to pull sufficient power from the pitch to access its inner memory and transmit its deposited information.The ATmega328P is the microcontroller that accepts the signal from the RFID reader in the form of buzzer which is the digital inputand we practice the driver ULN2803.

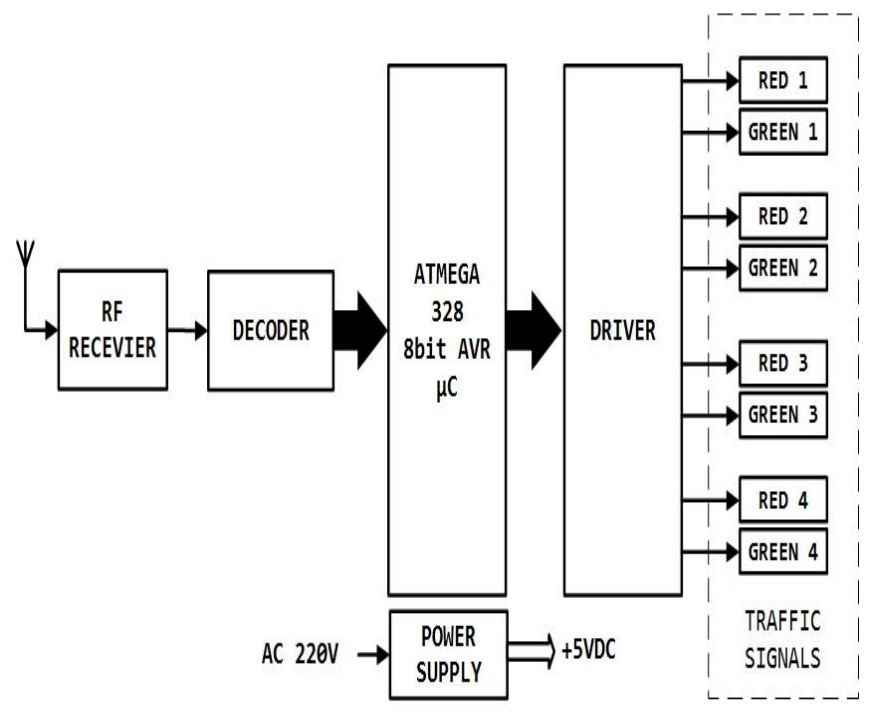

Fig.1. Block Diagram of Traffic Control System

It is a High voltage, high current Transistor Array IC used exclusively through microcontrollers which initiates high power loads. This IC comprises of a eight NPN Darlington coupled transistors with communal Clamp diodes for swapping the loads allied to the output. The output is the Led that is traffic lights. The ATmega328P is used has the microcontroller and the Arduino acts as a Programmer for the microcontroller.

RFID Reader it works on the concept called near field communication. It as a coil which acts like a transmitter and receiver. RFID Tag has a coil which is an antenna it transfers power and wireless transmission takes place. The
RFID reader reads the number present on the tag and an microcontroller inside the reader process it.

\section{FLOW CHART}

Sketch is used to write the code for the traffic control system. The Fig 2 shows the flow chart for the traffic light control. First initialization of the pin number and naming the pins are done. Next initial working of the traffic light for lane 1 is done by making the LED's high and low, for lane 1 Red 1 is made low and Green 1 is made high and for all the remaining LED's Red led is made high and green led low. This stage is made to remain for 5 seconds.

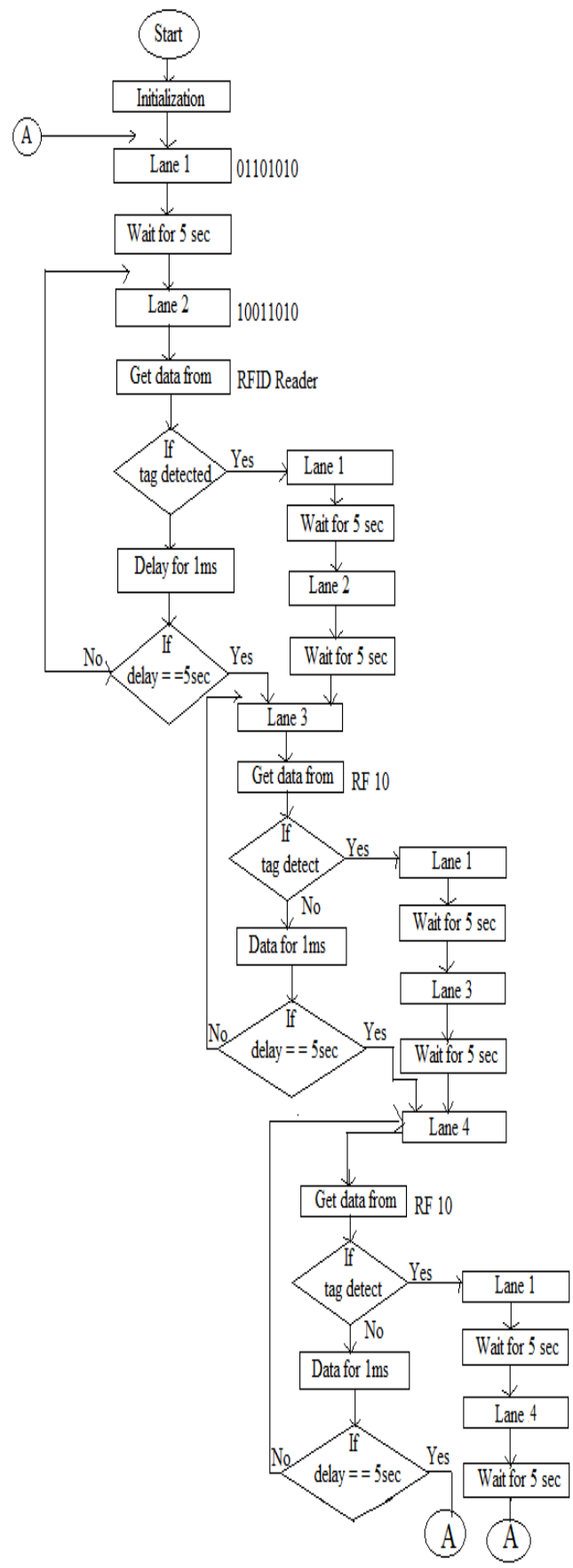

Fig. 2 Flow chart for Traffic Control System 
For Lane 2 the LED's Red2 is made low and Green 2 is made high and for all the remaining LED's Red led is made high and green led low. And now we read the data from RFID reader to check whether the Tag is detected if yes the tag is detected which means an ambulance is present in the lane and the traffic need to be cleared here we consider ambulance to be present only in lane 1 .

Therefore Lane 1 traffic is made clear by giving Red 1 is made low and Green 1 is made high and for all the remaining LED's Red led is made high and green led low for 5seconds. If no the delay of $1 \mathrm{msec}$ to change all the LED's to red and the Lane 2 traffic iscleared Red 2 is made low and Green 2 is made high and for all the remaining LED's Red led is made high and green led low for 5 seconds. The above procedure is continued for all other lanes.

\section{Ambulance Unit}

The ambulance unit consists of different sensors like heart beat sensor, body temperature sensor which $t$ is used to measure the heart rate and temperature of the body. As shown in Fig. 3. Three different keypad consoles placed, one is Blood Bank keypad console that is used to enter the blood group of the patient, and the other will be is Hospital keypad console used to enter the nearest hospital name.The android application constantly running on the mobile phone looks after the readings from the sensor and checks whether the temperature or heart beat goes beyond the normal rate [9].

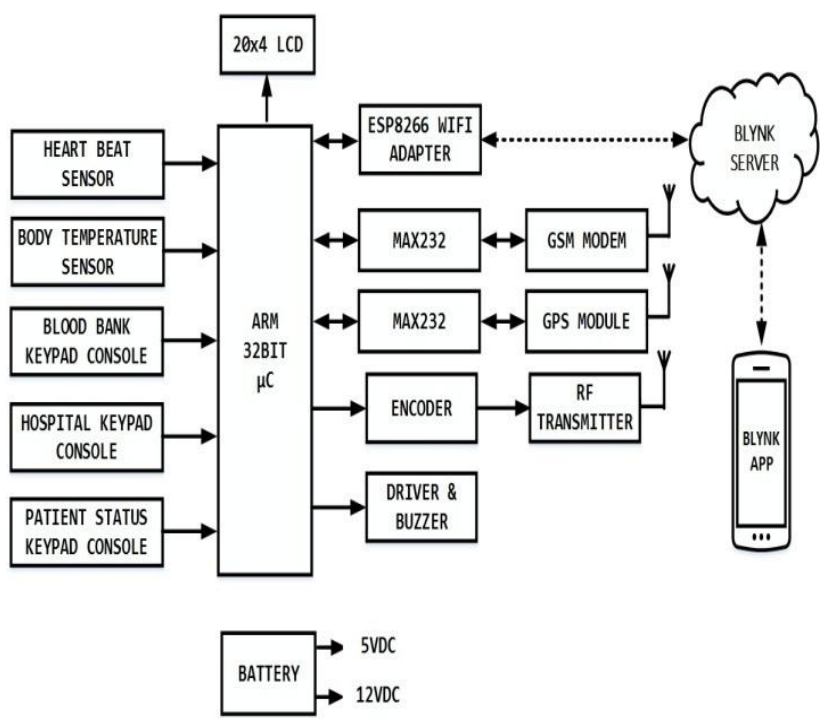

Fig. 3 Block Diagram of Ambulance Unit

so that the information of the patient will be sent to the nearest hospital, Next console is patient status keypad console this is used to enter the status of the patient whether it is a pregnancy patient or accident patient. All these details are sent to the ARM 32bit microcontroller and these details are displayed in 20x4 LCD. A ESP8266 Wi-Fi adapter is used to send the messages and information to the Blynk Server to the mobile through IoT. A GSM modem is used to send information to the mobile without using the internet connection. A GPS module gives the location of the ambulance. Radio Frequency transmitter is used to transmit the information about the arrival of the ambulance.

Ambulance unit the power is switched on and the code is uploaded to the Arduino due. The GSM MODULE, ESP8266, GPS Receiver and LCD Display is connected to the Arduino due board. The ESP8266 is connected to temperature sensor and heartbeat sensor. The temperature and heartbeat of the patient is measured and it is displayed in the LCD and in turn it is sent to the hospital and the blood bank. First of all me choose the nearby hospital that is displayed on the LCD screen. Later we select the type of patient, here we consider four types of patient heart attack, accident pregnant and other and further we select blood group if required and finally the condition of the patient whether critical, very critical, normal and other. These information of the patient is sent to the hospital via GSM module and blood bank gets information only if the situation is selected as critical or very critical. The location of the ambulance is also sent to the hospital and bloodbank has latitude and longitude information using GPS. All the above collected data is sent to hospital and bloodbank via SMS or Blynk app.

Initialization of the LCD display, GSM, GPS, ESP8266, Pulse sensor, Body Temperature sensor and Keypad are done by assigning pin numbers for the above considered parameters. After the initialization the different data are read from the ambulance unit that is ,the state of the patient is chosen based on four categories they are accident, pregnant, heart attack and others as well as patient blood groups like $\mathrm{O}, \mathrm{O}+, \mathrm{A}, \mathrm{A}+, \mathrm{B}, \mathrm{B}+, \mathrm{AB}-, \mathrm{AB}+$ is selected in case if needed. Meanwhile the patients Heartbeat and temperature is monitored.

We provide different hospitals in the options, based on the criteria of distance and availability of the doctor to the nearest hospital will be selected and the SMS will be directed.After collecting these data further the body temperature, pulse rate data are collected and the SMS to the respective hospitals about the patient details and location of the ambulance is sent and same details are sent to the blood bank and the traffic data is sent to the traffic light controller to clearance of the road. 


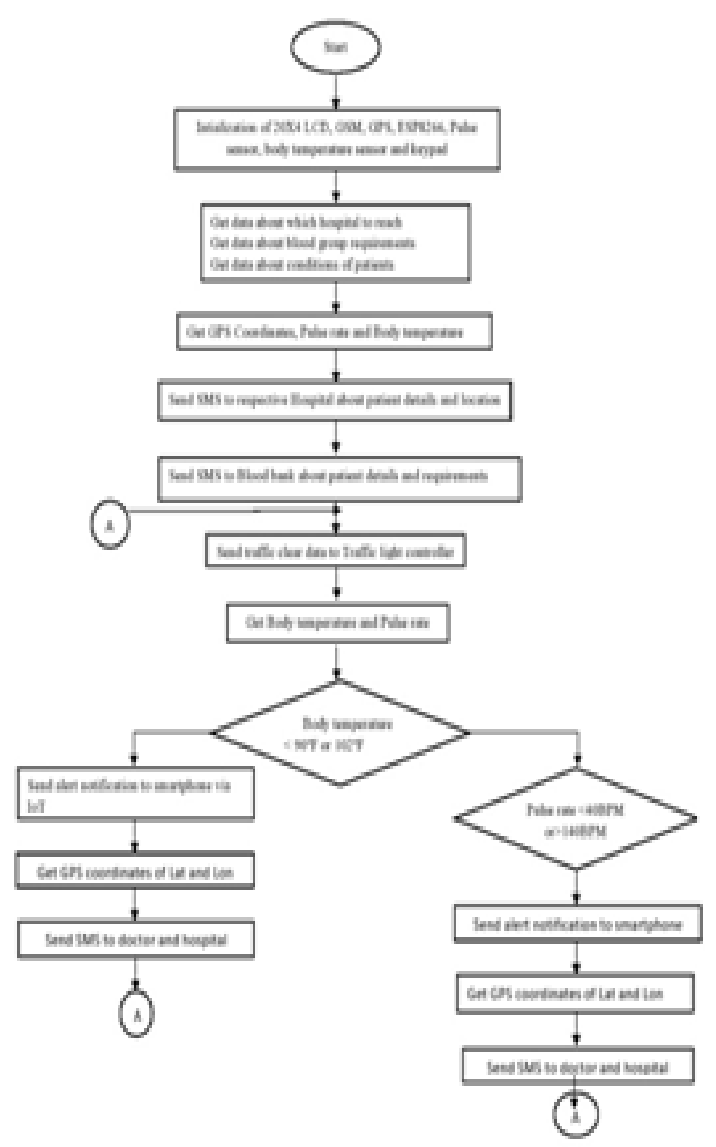

Fig. 4 Flowchart for Ambulance unit

If the body temperature is high consider above $90^{\circ} \mathrm{c}$ check for pulse rate and if its above $140 \mathrm{bpm}$ then send alert notification to smart phone via IoT, get GPS coordinates of latitude and longitude and send SMS to doctor and hospital. If body temperature is low then send alert notification to smart phone via IoT, get GPS coordinates of latitude and longitude and send SMS to doctor and hospital.

\section{RESULT}

The proposed paper describes two systems, patient wellbeing specialist care system and intellectual traffic control scheme. In health observing system, the patient's vital health constraints such as Heart Rate and Body Temperature, Blood pressure, Glucose Level are supervised and Blood Group is checked and the status of the patient is directed to the clinic for examination. In traffic control portion, RFID reader on the ambulance will converse with the $R F$ receiver fixed on the lane which is positioned $100 \mathrm{~m}$ away from the Traffic signal. Sketch is used for coding to control the traffic signals that automatically turns the red light to green when the ambulance is identified using the RFID reader. In this proposed Traffic control model we think through four lanes. Among the four lanes suppose if the ambulance is present in lanel then the traffic light in that particular lane changes to green if it is red in color. Rest of the lane the traffic light will be red in color. In the meanwhile health is monitored for every few minutes. Thus the traveltime of the ambulance to reach the hospital is reduced. Using IoT technology, the patient wellbeing is examined for each minute. Fig. 5 shows the normal condition of the ambulance when there is no ambulance near the traffic signal. Fig. 6 shows the change of signal when ambulance is $100 \mathrm{~m}$ away from the traffic signal. The Fig.7 shows the halt condition .Halt condition is the time gap for the signal to change from one state to another. This helps in avoiding clashing of vehicles due to quick change of signal state and hence traffic signal is controlled smoothly.

The information of the patient is sent to the hospital via GSM module and blood bank gets information only if the condition is chosen as critical or very critical as shown in Fig.8. The locality of the ambulance is also sent to thehospital and blood bank that has latitude and longitude information using GPS.

All the above collected data is sent to hospital and blood bank via SMS or Blynk app. the state of the patient is chosen based on four categories they are accident, pregnant, heart attack and others as well as patient blood groups like $\mathrm{O}, \mathrm{O}+, \mathrm{A}, \mathrm{A}+, \mathrm{B}, \mathrm{B}+, \mathrm{AB}-, \mathrm{AB}+$ is selected in case if needed. Meanwhile the patients Heartbeat and temperature is monitored. The nearest hospital will be selected and the SMS will be sent to the respective hospital and the blood bank.

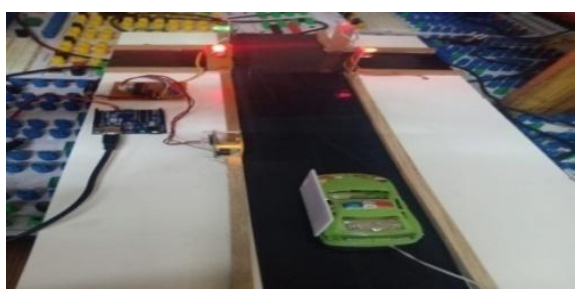

Fig. 5 Normal Traffic Condition

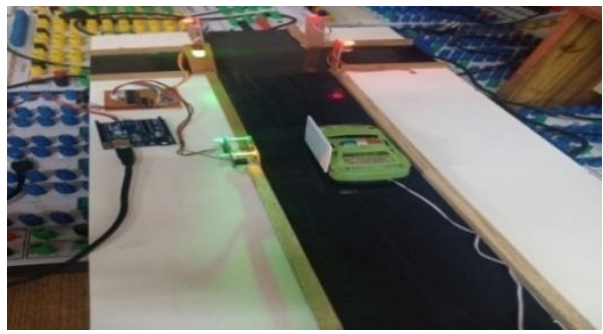

Fig. 6 Ambulance is sensed by RFID reader

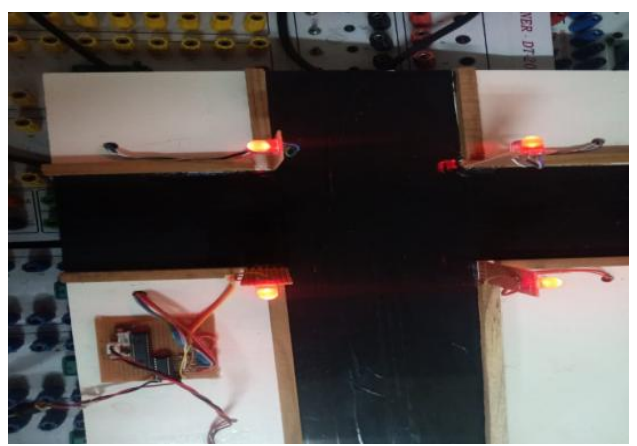

Fig. 7Halt condition

Published By:

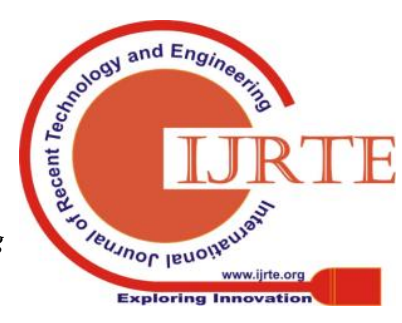




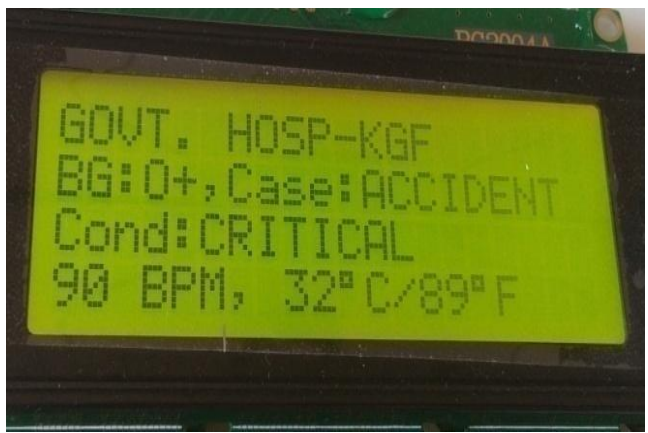

Fig. 8 Demonstration of Patient's Facts

The facts of the patient are directed over SMS to the Sick bay as well as Blood bank .The Fig.9 to Fig.12 demonstrates the SMS directing to Hospital, SMS directing to the Blood bank, SMS acknowledged by the hospital and the blood bank respectively.
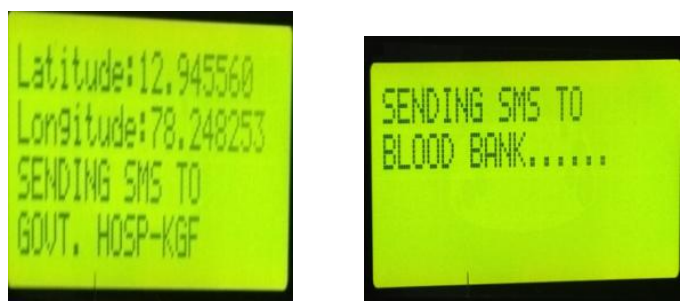

Fig.9 Directing SMS to hospital Fig.10 DirectingSMS to Blood bank

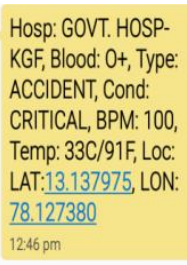

Fig.11 SMSacknowledged by the hospital Fig.12 SMSacknowledgedby the Blood bank

\section{CONCLUSION}

In the proposed method, a traffic control and smart ambulance system employed using RFID and IoT respectively. This hardware module can easily estimate the arrival of ambulance and clear the traffic. The method used for Smart Ambulance can be simply applied in an intellectual traffic control system in a massive populous nation. The aforementioned technique will perform a significant part in assessing the traffic control and provide the needed treatment for the patients in time. However it still requires improvement to accomplish greater precision.

\section{FUTURE SCOPE}

The proposed approach can be developed by using the selfregulatingPower Source that saves the power and via the GPRS map as an additional pace for improvement and pick out the preeminent road for the emergency conveyance. It can be implemented using Mat LAB by considering different parameters.

\section{REFERENCES}

1. Wenwen Kang et.al, "Traffic Signal Coordination for Emergency Vehicles", 2014 IEEE 17th International Conference on Intelligent Transport Systems (ITSC) October 2014, Qingdao China.

2. $\quad$ S. Pradeep Kumar et.al, "Call Ambulance Smart Elderly Monitoring System with Nearest Ambulance Detection using Android and Bluetooth", 2016 IEEE Second International Conference on Science and Technology Engineering and Management.

3. G.Beri and P.Ganjare "Intelligent ambulance with traffic control" 3rd National Conference on Advancements in Communication, Computing and Electronic Technology[ACCET-2016] held at M.E.S College of Engineering, Pune 11-12, February 2016.

4. Rajeshwari S et.al, "Implementing Intelligent Traffic Control System for Congestion Control, Ambulance Clearance and Stolen Vehicle Detection",2014 IEEE Sensors Journals.

5. OmkarUdawantet.al, "Smart Ambulance System using IoT", International Conference on Big Data, IoT and Data Science (BID)Vishwakarma Institute of Technology, Pune, Dec 2017

6. Liang Qi et.al, "A Two-level Traffic Light Control Strategy for Preventing Incident-Based Urban Traffic Congestion”,2016 IEEE transactions on Intelligent Transport Systems.

7. Abdullahi Chowdhury et.al,"Priority Based and Secured Traffic Management System for Emergency Vehicle using IoT", Faculty of Science and Technology Federation University Australia 2015.

8. SabeenJavaid et.al, "Smart Traffic Management System using Internet of Things", 2018 20th International Conference on Advanced Communication Technology (ICACT).

9. HoomanSamani et.al, "Robotic Automated External of the Defibrillator Ambulance for Emergency Medical Service in Smart Cities",2015 IEEE Access Journal.

10. Ahmed, Syed Thouheed, M. Sandhya, and Sharmila Sankar. "A Dynamic MooM Dataset Processing Under TelMED Protocol Design for QoS Improvisation of Telemedicine Environment." Journal of medical systems 43, no. 8 (2019): 257

11. Ahmed, Syed Thouheed, M. Sandhya, and Sharmila Sankar. "An Optimized RTSRV Machine Learning Algorithm for Biomedical Signal Transmission and Regeneration for Telemedicine Environment." Procedia Computer Science 152 (2019): 140-149.

12. Patil, Kiran Kumari, and Syed Thouheed Ahmed. "Digital telemammography services for rural India, software components and design protocol." In 2014 International Conference on Advances in Electronics Computers and Communications, pp. 1-5. IEEE, 2014

13. Thouheed, Syed, S. Ahmed, M. Sandhya, and S. Shankar. "ICT's Role in Building and Understanding Indian Telemedicine Environment: A Study." In Information and Communication Technology for Competitive Strategies. Lecture Notes in Networks and Systems, vol 40. Springer Singapore, 2019. 